\title{
Transients in porous media: exact and modelled time-domain Green's functions
}

\author{
J. Kergomard*(a), D. Lafarge (b), J. Gilbert (b) \\ (a) LMA, CNRS, UPR 7051, Aix-Marseille Univ, Centrale Marseille, \\ F-13402 Marseille Cedex 20, France \\ (b) LUNAM Université, CNRS, UMR 6613, \\ Laboratoire d'Acoustique de l'Université du Maine, Avenue Olivier Messiaen, \\ 72085 Le Mans Cedex 9, France.
}

June 18, 2021

\begin{abstract}
Time domain responses of porous media have been studied by some authors, but generally the possible descriptions are given in the frequency domain. The aim of this paper, limited to materials with rigid skeleton considered as equivalent fluids, is to compare in time domain different descriptions by Johnson-Allard $(J A)$ as well as by Pride-Lafarge $(P L)$, with: $i$ ) some analytical approximate formulas based upon asymptotic high-frequency expansion ; ii) the exact formula by Zwikker and Kosten for the case of cylindrical pores. The paper starts with a construction analysis of the models $(J A$ and $P L)$. Then, the Green's function in the time domain is defined, written in scaled form, and shown to exhibit interesting properties of materials. In particular, a so-far overlooked decay length $\mathcal{L}$ describing a high-frequency attenuation-without-distortion effect is identified in terms of Brown's tortuosity, Johnson's and Allard's known characteristic viscous and thermal lengths, and two, unknown in general, characteristic viscous and thermal surfaces. The numerical computation of the Green's function is done by FFT, with some precautions, because of the importance of the higher frequencies on the response shape: the substraction of the diffusive (low frequencies) approximation largely improves the results of the FFT. The $P L$ description is shown to be the best full-frequency general model with remaining small discrepancies due to unsatisfactory account of the mentioned surface-parameters.
\end{abstract}

Keywords: Pulse propagation; Transient signals; Porous material

PACS: $43.55 \mathrm{Rv}, 43.55 \mathrm{Ti}, 43.20 \mathrm{Gp}, 43.20 \mathrm{Bi}, 43.20 \mathrm{Hq}$

\section{Introduction}

\footnotetext{
${ }^{*}$ Tel 33 491164381, Fax 33 491228248, kergomard@lma.cnrs-mrs.fr
} 
Linear wave propagation in homogeneous porous media saturated with a viscothermal fluid such as ambient air, has been the subject of extensive research. Traditionally, for the case when the wavelength is large compared to the pore size, it has been described on the basis of the so-called two-scale asymptotic homogenization theory [1]- 8]. For materials with skeleton sufficiently heavy and/or rigid to be motionless, this leads to an effective medium theory in which the medium permittivities are two linear operators, one representing an effective density and the other an effective compressibility [9, 10. These operators, while nonlocal in time as a result of delayed responses due to the losses (temporal dispersion), are given as local in space by the homogenization process at the dominant order. In other words, no spatial dispersion arises, so that the response of the material at a given macroscopic point (physically, an elementary coarse-graining volume) is determined by the history of the pertinent field variables at the given point but not the neighboring points. As such, this description is a special case not directly applicable to all geometries. It does not describe the situations where structures in the form of Helmholtz resonators are present1. Nevertheless, most of the materials used in noise control for sound absorption, do not present in their inner structure very different pores sizes. In this paper we explicitly assume the absence of very different pores sizes, automatically ensuring at long wavelengths the validity of the usual two-scale asymptotic homogenization.

It gives us explicit recipes to compute from microgeometry the effective density and compressibility, complex functions of frequency but not of wavenumber, in the above framework. In practice, the computation is not possible in full detail; it is however not required to be done to arrive at a relatively precise description. In absence of a complete knowledge of the microgeometry, a widely used semi-phenomenological model which depends on a small set of independently measurable geometrical parameters of the structure, is given by the well-known formula of Johnson et al. 17 for the density, and likewise, the Champoux-Allard [18] or the Lafarge et al. [10] formula for the compressibility? These approximate expressions (denoted $J A$ ) of the two constitutive functions, essentially are the result of:

1) an exact description of the high-frequency limits of the two functions, density and compressibility, in terms of the concepts of ideal-fluid tortuosity $\alpha_{\infty}$ and characteristic lengths $\Lambda\left[17\right.$ and $\Lambda^{\prime}[18$, the viscous and thermal relaxation processes being 'frozen' in this limit4,

2 ) an exact description of the low-frequency limits, in terms of the concepts of d.c. viscous and thermal permeabilities, $k_{0}\left[19\right.$ and $k_{0}^{\prime}$ [20, the viscous and

\footnotetext{
${ }^{1}$ In this case, if the microgeometry splits in parts with pore sizes sufficiently different to imply different rescalings of the microscopic governing equations in the different parts, a solution can still be written using the principle of asymptotic homogenization [1]. Independently, a general nonlocal theory of propagation along a symmetry axis in macroscopically homogeneous materials having arbitrary microgeometry, has been recently proposed by one of the authors 12. Finally, different high-frequencies extensions of the idea of two-scale homogenization have also been introduced recently [13]- 16]. All of these extensions lie outside the scope of the present paper, as defined next.

${ }^{2}$ This excludes resonators, as in a resonator the neck and cavity dimensions differ by one order of magnitude at least.

${ }^{3}$ This last formula relies on a description of elastic-thermal effects entirely analogous to Johnson's description of inertial-viscous effects.

${ }^{4}$ In the frozen limit the viscous and thermal relaxation processes have no time to develop; in the relaxed limit they have enough time to fully develop.
} 
thermal relaxation processes being 'relaxed' in this limit, and finally,

3) an assumption that these frozen and relaxed limits 1) and 2) are connected in the simplest reasonable manner, i.e. by means of the simplest 'relaxation' functions of frequency $\omega$ having their singularities and zeros lying on the imaginary half-axis in the complex $\omega$ plane (see [17] Appendix A, and 20] or [10] Appendix C).

That the singularities are on a half imaginary axis is a mathematical expression of the fact that we actually restrict to the class of materials allowing to apply the direct two-scale homogenization process. The pure causality condition would only require the singularities to be in a half plane. Our stronger assumption may be rephrased physically by saying that the fluid velocity pattern at the pore scale is divergence-free for the purpose of the determination of the density, and the pressure pattern is uniform for the purpose of the determination of the compressibility; or in essence, that the operators are local operators in space, at long wavelengths, in the geometries we consider. The remaining assumption that the functions are the simplest reasonable 'relaxation' ones, consists in making the additional but adjacent assumption that the considered geometries manifest a relatively narrow - not bimodal, for example - distribution of pore sizes. Then the distribution of poles on the imaginary axis is simple and the whole pattern of response functions on the real axis is strongly determined by the low- and high-frequency behaviors.

In this manner, a simple, resp. viscous and thermal, relaxation-transition description of the density and compressibility functions is obtained, that may be thought to be well-verified in a wide class of materials as long as the wavelengths are large compared to the typical dimensions of the coarse-graining averaging volumes. At this point, we may mention a similar relaxation-transition approach, developed by Wilson [21,22. In Wilson's approach, the emphasis is not made on the low-frequency and high-frequency limits, but directly on the transition. The quality of the description obtainable with Wilson's models is comparable to that of model $J A$, but the parameters become purely adjustable parameters not precisely defined in terms of the microgeometry, and not clearly obtainable by non-acoustical means.

Subsequently, the low-frequency relaxed limit 2) was made more precise by Pride et al. 23] and Lafarge 20 (the next viscous and nontrivial thermal terms now being exactly described thanks to the introduction of the additional notions of d.c. viscous and thermal tortuosities $\alpha_{0}$ and $\alpha_{0}^{\prime}$ ), resulting in a slightly improved (denoted $P L$ ) description of the relaxation transitions of the two functions. Notice that some confusions were present in the original works 23] and 20 (not readily available) and other subsequent ones (e.g. 24]), so that the presentation we give later on, of the $P L$ description, may be worthwhile.

In Ref. 25, Fellah et al. concentrated on the time-domain expression of the high-frequencies asymptotics implied by this mode 5 . Expanding, in the highfrequency limit, the $P L$ density and compressibility in powers of the inverse Stokes number $S_{T}^{-1}$ (defined as the ratio between boundary layer thickness and characteristic pore size), and retaining the (exact) zero and first order terms and the (model-dependent) second order terms, they derived an asymptotic time-domain pressure wave equation. Using fractional-derivative and Laplace-

\footnotetext{
[38.

${ }^{5} \mathrm{~A}$ short, non exhaustive list of works concerned with the time-domain description is: [26]-
} 
transform calculus, they were able to solve this equation in elegant manner through the calculation of a corresponding asymptotic Green's function of the unbounded medium. Recall that a Green's function or impulse response, as a function of time, extends and flattens when observed at fixed locations more and more remote from the spatial point where it originates.

Now, the results of the calculations made in 25 seem to indicate that the terms of second order yield a significant effect on the Green's function for resistive porous materials, and, in addition, that the effect is mainly an effect on the amplitude without noticeable distortion of the time wave pattern (see Figs. 2 and 3 of paper [25]).

In the present paper, our purpose is threefold.

First, we wish taking advantage of recent clarifications regarding nonlocal (spatial dispersion) effects 12 to propose a lucid review of the above local (frequency dispersion) theory, stressing the physical hypotheses behind it. By recalling the construction principle of the models $J A$ and $P L$, we make clear that these models a priori lead to inaccurate descriptions of the second order highfrequency terms $S_{T}^{-2}$. This is highlighted on the simple example of cylindrical circular tubes. This is the matter of section 2 ,

Next, we give a very simple analytic derivation of the attenuation-withoutdistortion finding of Ref. 25] summarized above. Indeed, generalizing to arbitrary geometry a work done by Polack et al. 39. for cylindrical circular tubes, we show the following exact property (for 1D propagation along one axis $x$ ):

$$
G_{o(2)}(x, t)=G_{o(1)}(x, t) \exp (-x / \mathcal{L}),
$$

where $G_{o(1)}(x, t)$ and $G_{o(2)}(x, t)$ are the two first asymptotic Green's functions computed by retaining in the wavenumber the terms up to the first and second order on the inverse Stokes number $S_{T}^{-1}$ respectively. This is done in section 3. using a scaled-form of the Green's function. The first is only determined by $\alpha_{\infty}, \Lambda$ and $\Lambda^{\prime}$, and exactly predicted by the models $J A$ and $P L$. The second demonstrates the mentioned attenuation-without-distortion effect through the exponential. The decay length $\mathcal{L}$, however, depends in part of the next frozen parameters, two viscous and thermal purely geometrical characteristic surfaces $\Sigma$ and $\Sigma^{\prime}$ involved in the above-mentioned terms $S_{T}^{-2}$ :

$$
\mathcal{L}=\frac{2 \Lambda^{2} c_{f}}{\nu \sqrt{\alpha_{\infty}}}\left[\frac{3 \Lambda^{2}}{\Sigma}-1+\frac{2(\gamma-1) \Lambda}{\Lambda^{\prime} \sqrt{\operatorname{Pr}}}+\frac{(\gamma-1) \Lambda^{2}}{\Lambda^{\prime 2} \operatorname{Pr}}\left(\frac{3 \Lambda^{\prime 2}}{\Sigma^{\prime}}-3-\gamma\right)\right]^{-1},
$$

where the fluid constants are, $\nu=\eta / \rho_{f}$ the fluid kinematic viscosity, $c_{f}=$ $\sqrt{K_{f} / \rho_{f}}$ the adiabatic speed of sound, Pr the Prandtl number, and $\gamma=c_{p} / c_{v}$ the ratio of heat coefficients. Now, because the models $J A$ and $P L$ (especially $J A$ ) give inaccurate predictions for the $S_{T}^{-2}$ terms, this decay length $\mathcal{L}$ will not be accurately captured by the models. Indeed, on the simple example of cylindrical circular tubes it can be checked that $\mathcal{L}$ is completely misrepresented by model $J A$, which gives a negative estimate for it, and still largely underestimated by model $P L$, which produces only about $1 / 3$ of its correct value, due to $50 \%$ overestimation of surfaces $\Sigma$ and $\Sigma^{\prime}$. Both models $J A$ and $P L$ do not describe the correct high-frequency attenuation-without-distortion effect. Technical details are given in Appendix to lighten the main text.

Finally, our last objective is to show that, in spite of its faulty description of 
the decay length $\mathcal{L}(2)$, the model $P L$, nevertheless furnishes a relatively precise description of the complete exact Green's function, especially when compared to other formulas. The asymptotic analytic Green's function $G_{o(2)}$ provides a reasonable description of the complete Green's function with the same number of parameters as $J A$, but it uses parameters $\Sigma$ and $\Sigma^{\prime}$ which are unknown in general. The merits and drawbacks of the different descriptions are illustrated on the example of circular pores - i) computing the exact Green's function through FFT and the known Zwikker and Kosten full frequency formulas; ii) computing likewise the model Green's functions $J A$ and $P L$ through FFT; iii) computing the exact asymptotic Green's function $G_{o(2)}$ through (11-2) with the known exact values of the involved parameters (namely $\alpha_{\infty}=1, \Lambda=\Lambda^{\prime}=R$, $\Sigma=\Sigma^{\prime}=R^{2}$ ); and vi) computing the 'model asymptotic' Green's functions through (112) with the model values of the involved parameters $\left(\alpha_{\infty}=1, \Lambda=\right.$ $\Lambda^{\prime}=R$, and $\Sigma=\Sigma^{\prime}=\frac{3}{2} R^{2}$ for $P L$ and $\Sigma=\Sigma^{\prime}=\infty$ for $J A$ ). The results of FFT computation are given in section 4 for the full-frequency models, while the results for asymptotic expansions are given in Appendix.

\section{Basic equations}

We start by recalling the form of the macroscopic equivalent-fluid equations in the frequency domain (see $10,17,40,41]$ ):

$$
\rho_{f} \alpha(\omega) i \omega v_{i}=\nabla_{i} p ; K_{f}^{-1} \beta(\omega) i \omega p=\nabla . v,
$$

where by definition, $\mathbf{v}$ and $p$ are the macroscopic velocity and pressure obtained by coarse-graining (averaging) the microscopic fluid velocity and pressure fields, $-i \omega$ is the time derivative, $\rho_{f}$ and $K_{f}$ are the saturating-fluid density and adiabatic bulk modulus, and $\alpha(\omega)$ and $\beta(\omega)$ are the dynamic tortuosity and the dynamic compressibility. Notice that for simplicity, isotropy or 1D propagation along one principal axis is assumed, so that $\alpha(\omega)$ is a scalar.

Eqs. (3) make apparent the frequencies but not the wavenumbers associated to the time- and space-variable fields. This is consistent with the hypothesis that the effects of spatial dispersion are negligible. A necessary - but not sufficient - condition for this, is that the wavelengths are large. The complementary condition which will ensure that spatial locality is verified, is that no very different pore sizes are present, so that in particular, the presence of structures in the form of Helmholtz resonators is excluded. Indeed, with resonators, the fluid exchanged to and fro, is always associated by mass conservation with a corresponding spatial inhomogeneity in the wavefield. The resonance cannot occur without concomitant spatial inhomogeneity in the macroscopic fields. As such, it is an effect of spatial dispersion, see 42 p. 360, no matter how large the wavelengths actually are. Now, if we restrict sufficiently the possible geometries, resonances cannot occur and the long-wavelength propagation becomes practically devoid of spatial dispersion i.e. is given by the traditional homogenization.

The application of the asymptotic two-scale homogenization then yields two different microscopic boundary value formal problems to be solved for determining the two constitutive functions $\alpha(\omega)$ and $\beta(\omega)[10$.

The first action-response problem specifies the (velocity) response of a viscous incompressible fluid subject to an applied time harmonic, spatially uniform 
bulk force source term:

$$
\begin{aligned}
\frac{-i \omega \rho_{f}}{\eta} \mathbf{w} & =-\nabla \Pi+\nabla^{2} \mathbf{w}+\mathbf{e}, \\
\nabla \cdot \mathbf{w} & =0
\end{aligned}
$$

in the pore space (with $\Pi$ a periodic or stationary random field, in periodic or stationary random geometries), and satisfying on the pore surface (no slip condition),

$$
\mathbf{w}=\mathbf{0} .
$$

In this problem, $\mathbf{e}$ is a dimensionless unit vector in the direction of the applied bulk force, and $\mathbf{w}$ is the scaled response velocity field (dimension of length ${ }^{2}$ ). It determines the dynamic permeability $k(\omega)$ and dynamic tortuosity $\alpha(\omega)$ introduced in the landmark paper by Johnson et al. 17, by the relations:

$$
k(\omega)=\frac{\eta \phi}{-i \omega \rho_{f} \alpha(\omega)}=\langle\mathbf{w}\rangle \cdot \mathbf{e},
$$

where \langle\rangle is the coarse-graining averaging operation in the pore space, and $\phi$ is the porosity (specific connected pore volume).

The second action-response problem specifies the (excess temperature) response of a thermal fluid subject to an applied time harmonic, spatially uniform pressure source term:

$$
\frac{-i \omega \operatorname{Pr} \rho_{f}}{\eta} \theta=\nabla^{2} \theta+1
$$

in the pore space, and satisfying on the pore surface (no temperature-jump condition 6 ),

$$
\theta=0 .
$$

Here, 1 is a dimensionless unit constant representing the applied pressure, and $\theta$ is the scaled response excess temperature field (dimension of length ${ }^{2}$ ). It determines the functions $k^{\prime}(\omega)$ and $\alpha^{\prime}(\omega)$, thermal counterparts of functions $k(\omega)$ and $\alpha(\omega)$ introduced by Lafarge [10,20], and then, the effective compressibility $\beta(\omega)$, by the following relationships:

$$
k^{\prime}(\omega)=\frac{\eta \phi}{-i \omega \operatorname{Pr} \rho_{f} \alpha^{\prime}(\omega)}=\langle\theta\rangle ; \beta(\omega)=\gamma-\frac{\gamma-1}{\alpha^{\prime}(\omega)}
$$

where $\gamma$ is the ratio of the specific heats.

The above action-response problems and response-factor identifications (7) and (10) are written in blind manner by applying the traditional two-scale asymptotic homogenization. As we have insisted, it makes the important assumption that it is possible to neglect spatial dispersion. This is manifested in the first problem by the force source term $\mathbf{e}$ which is set to a spatial constant (simultaneously, the velocity field $\mathbf{v}$ is represented by a divergence-free field), and in the second problem by the pressure source term 1 which is also set to a spatial constant (gradient-free)7. Indeed, we could have written these prob-

\footnotetext{
${ }^{6}$ The solid specific volume $(1-\phi)$ is assumed sufficiently important to ensure that the specific fluid-solid mass ratio is small; then the solid is inert thermally and remains at ambient temperature.

${ }^{7}$ In fact, what is really meant here is that in this second problem, locally, the pressure field
} 
lems and identifications directly without using the homogenization process, on the sole basis of assuming that spatial dispersion effects are absent. Now, as a result of the divergence-free and gradient-free nature of velocity and pressure in the given action-response problems, it can be shown that the functions $k(\omega)$ and $k^{\prime}(\omega)$ have purely imaginary singularities [17], [10], and thus are smooth functions on the real axis - this is the point 3) mentioned in Introduction. This crucial point in the construction of the models will be considered at more length in section 2.2 .

\subsection{Frozen and relaxed limits}

In absence of a complete information on the microgeometry, the two abovementioned microscopic formal boundary-value problems cannot entirely be worked out and their exact detailed solutions (from which $\alpha(\omega)$ and $\beta(\omega)$ can in principle be extracted by coarse-graining, see Eqs.(7) and (10) ) are missing. Nevertheless, in the limit of high-frequencies and low-frequencies, some general characteristics of the solutions and corresponding functions $\alpha(\omega)$ and $\beta(\omega)$ may be obtained. That may be sketched as follows.

\subsubsection{High frequencies (frozen limit)}

In the limit of high frequencies, the viscous and thermal terms $\nabla^{2} \mathbf{w}$ and $\nabla^{2} \theta$ become negligibly small compared to the other terms. The fluid motions, except for vanishingly small viscous and thermal boundary layers at the pore walls, become close to those of an inviscid nonconducting fluid $(\eta=0, \kappa=0$, with $\kappa$ the thermal conductivity $\left.c_{p} \eta / \mathrm{Pr}\right)$. We refer to this limit simply as the 'frozen limit' (see footnote 4 ). Accordingly, the quantities $\frac{-i \omega \rho_{f}}{\eta} \mathbf{w}$ and $\frac{-i \omega \operatorname{Pr} \rho_{f}}{\eta} \theta$ everywhere tend (except at the pore walls) to the 'frozen' fields $\mathbf{E}$ and $I$ verifying the following equations in the pore space, (with $\varphi$ a periodic or stationary random field, in periodic or stationary random geometries),

$$
\begin{aligned}
\mathbf{E} & =-\boldsymbol{\nabla} \varphi+\mathbf{e} ; I=1, \\
\boldsymbol{\nabla} \cdot \mathbf{E} & =0,
\end{aligned}
$$

and verifying at the pore walls ( $\mathbf{n}$ is the normal on the latter),

$$
\mathbf{E} \cdot \mathbf{n}=\mathbf{0}
$$

Now assume, following Johnson et al. 17 and Allard 40, that the poresurface interface appears locally plane in this asymptotic high-frequency frozen limit. This is in principle an assumption that the viscous and thermal boundary layer thicknesses $\delta=\sqrt{\frac{2 \eta}{\rho_{f} \omega}}$ and $\delta^{\prime}=\sqrt{\frac{2 \eta}{\rho_{f} \omega \operatorname{Pr}}}$ eventually become small compared to a characteristic radius of curvature of the pore surface 8 . Then, the functions $\alpha(\omega)$ and $\alpha^{\prime}(\omega)$ expand in integral power series of these thicknesses,

may be viewed as having a uniform gradient; but the pressure linear variation around the mean may be omitted in a coarse graining volume, as it has no effect on the mean temperature. As soon as spatial dispersion is introduced, it no longer makes sense to represent the source terms by spatial constants. The spatial inhomogeneity of the source terms must be considered.

${ }^{8}$ In practice this is not strictly necessary: because of the smooth nature of response functions, the limiting behaviors (14) become meaningful much more rapidly. 
which allows us writing a prior, 9

$$
\begin{aligned}
\alpha(\omega) & =\alpha_{\infty}+\frac{2 \alpha_{\infty}}{\Lambda}\left(\frac{\eta}{-i \omega \rho_{f}}\right)^{1 / 2}+\frac{3 \alpha_{\infty}}{\Sigma}\left(\frac{\eta}{-i \omega \rho_{f}}\right)+O\left(\frac{1}{-i \omega}\right)^{3 / 2} ; \\
\alpha^{\prime}(\omega) & =\alpha_{\infty}^{\prime}+\frac{2 \alpha_{\infty}^{\prime}}{\Lambda^{\prime}}\left(\frac{\eta}{-i \omega \rho_{f} \mathrm{Pr}}\right)^{1 / 2}+\frac{3 \alpha_{\infty}^{\prime}}{\Sigma^{\prime}}\left(\frac{\eta}{-i \omega \rho_{f} \mathrm{Pr}}\right)+O\left(\frac{1}{-i \omega}\right)^{3 / 2}(14)
\end{aligned}
$$

The geometrical parameters $\alpha_{\infty}$ and $\alpha_{\infty}^{\prime}$ (dimensionless) and $\Lambda$ and $\Lambda^{\prime}$ (dimension of length) must be some pore averages constructed with the frozen fields $\mathbf{E}$ and $I$. The next order geometrical parameters $\Sigma$ and $\Sigma^{\prime}$ (dimension of surface) are dependent on other fields involved in the asymptotic limit and have not been worked out so far. Detailed calculations made by Johnson and Allard 17, 18, to which we refer the reader, show that the parameters $\alpha_{\infty}, \alpha_{\infty}^{\prime}, \Lambda$ and $\Lambda^{\prime}$ may be written as follows (see also, in the most detailed manner for $\Lambda$, [43]):

$$
\frac{1}{\alpha_{\infty}}=\langle\mathbf{E}\rangle \cdot \mathbf{e}=\frac{\langle\mathbf{E}\rangle^{2}}{\left\langle\mathbf{E}^{2}\right\rangle} ; \frac{1}{\alpha_{\infty}^{\prime}}=\langle\mathbf{I}\rangle=1,
$$

and,

$$
\frac{2}{\Lambda}=\frac{\int_{S_{p}} \mathbf{E}^{2} d S}{\int_{V_{f}} \mathbf{E}^{2} d V} \quad ; \quad \frac{2}{\Lambda^{\prime}}=\frac{\int_{S_{p}} I^{2} d S}{\int_{V_{f}} I^{2} d V}=\frac{\int_{S_{p}} d S}{\int_{V_{f}} d V},
$$

where $S_{p}$ denotes the pore walls and $V_{f}$ denotes the connected pore volume.

Parameter $\alpha_{\infty} / \phi$ is Brown's electric formation factor [44] (E represents either an inviscid-fluid scaled velocity or acceleration field for the problem of incompressible inviscid fluid flow, accelerating under the action of external bulk force or applied pressure drop, or else, an electric scaled field for the problem of electrical conduction in the bulk fluid [45], [46]). Parameter $\Lambda$ is an effective pore radius for dynamically connected pore sizes which was introduced by Johnson et al. 47. for the problem of electrical conduction in the bulk fluid, perturbed by a thin, different conducting layer at the pore walls. Parameter $\Lambda^{\prime}$ is a length characterizing a simpler effective pore radius - twice the fluid-volume to fluid-surface ratio - sometimes referred to as the Kozeny-Carman radius; Allard [18 identified it as the thermal counterpart of parameter $\Lambda$. An incomplete reasoning to obtain the parameter $\Lambda$, leading to an incomplete expression $2 / \Lambda=\int_{S_{p}} \mathbf{E} \cdot \mathbf{e} d S / \int_{V_{f}} \mathbf{E}^{2} d V$, is often made, e.g. [6] [48] [49]; the reasoning inaccuracy 10 was clarified and corrected in 43 , along a line tentatively sketched in [45] (Appendix D).

\subsubsection{Low frequencies (relaxed limit)}

In the opposite relaxed limit of low frequencies, the viscous and thermal terms $\nabla^{2} \mathbf{w}$ and $\nabla^{2} \theta$ eventually become much greater than the inertial terms $\frac{-i \omega \rho_{f}}{\eta} \mathbf{w}$

\footnotetext{
${ }^{9}$ Notice that the case of fractal geometry which modifies the exponent $1 / 2$ in the first correction terms - see [17 - is excluded by the assumption that the pore walls appear locally flat at the scale of the boundary layer thickness; the presence of sharp edges which modifies the exponent 1 in the second correction terms - see [43] - is also excluded by this assumption.

${ }^{10}$ The ignorance, in the bulk, i.e. outside the viscous boundary layer or the perturbed conducting layer, of a perturbation contribution due to a perturbed ideal-fluid or electrical bulk flow field orthogonal to the leading bulk flow field E, and having, contrary to the latter, nonzero normal components at the pore walls.
} 
and $\frac{-i \omega \operatorname{Pr} \rho_{f}}{\eta} \theta$ and the boundary layers extend to the whole fluid. Accordingly, the fields $\mathbf{w}$ and $\theta$ everywhere tend to the d.c. - or 'relaxed' - velocity and excess temperature fields $\mathbf{w}_{0}$ and $\theta_{0}$ verifying, in the pore space (with $\Pi_{0}$ a periodic or stationary random field, in periodic or stationary random geometries),

$$
\begin{aligned}
\boldsymbol{\nabla}^{2} \mathbf{w}_{0} & =\boldsymbol{\nabla} \Pi_{0}-\mathbf{e} ; \boldsymbol{\nabla}^{2} \theta_{0}=-1 \\
\boldsymbol{\nabla} \cdot \mathbf{w}_{0} & =0
\end{aligned}
$$

and verifying, at the pore walls,

$$
\mathbf{w}_{0}=\mathbf{0} ; \theta_{0}=0 .
$$

In this limit the functions $\alpha(\omega)$ and $\alpha^{\prime}(\omega)$ expand in Laurent's series,

$$
\alpha(\omega)=\frac{\eta \phi}{-i \omega \rho_{f} k_{0}}+\alpha_{0}+O(-i \omega) ; \alpha^{\prime}(\omega)=\frac{\eta \phi}{-i \omega \rho_{f} \operatorname{Pr} k_{0}^{\prime}}+\alpha_{0}^{\prime}+O(-i \omega) .
$$

The two first intervening geometrical parameters, $k_{0}$ (Darcy's viscous permeability) and $k_{0}^{\prime}$ (its thermal counterpart [10 20] ) on one hand, both having dimension of length ${ }^{2}$, and $\alpha_{0}$ (viscous tortuosity) and $\alpha_{0}^{\prime}$ (its thermal counterpart) on the other hand, both being dimensionless, are pore averages constructed with the relaxed fields $\mathbf{w}_{0}$ and $\theta_{0}$. Indeed, simple calculations show that they may be written [9, 20],

$$
\begin{aligned}
& k_{0}=\phi\left\langle\mathbf{w}_{0}\right\rangle \cdot \mathbf{e} ; k_{0}^{\prime}=\phi\left\langle\theta_{0}\right\rangle, \\
& \alpha_{0}=\frac{\left\langle\mathbf{w}_{0}^{2}\right\rangle}{\left\langle\mathbf{w}_{0}\right\rangle^{2}} ; \alpha_{0}^{\prime}=\frac{\left\langle\theta_{0}^{2}\right\rangle}{\left\langle\theta_{0}\right\rangle^{2}} .
\end{aligned}
$$

Mention that, among other things it is possible to show that $9,20,44,50$, whatever the geometry,

$$
\begin{aligned}
\alpha_{0}>\alpha_{\infty} & \geq 1, \\
k_{0} \leq k_{0}^{\prime} ; \alpha_{0} \geq \alpha_{0}^{\prime} & >1 ; \Lambda \leq \Lambda^{\prime},
\end{aligned}
$$

the equalities being satisfied only for the case of aligned cylindrical pores.

\section{$2.2 \quad$ Full-frequency models}

As explained before, it is only for sufficiently simple geometries that the assumed long-wavelength nature of the considered fields, automatically imply that the microscopic flow-field can be considered divergence-free for the purpose of determining the density (Eq. (5)), and likewise, the excess pressure field can be considered gradient-free for the purpose of determining the compressibility.

Now, Johnson et al. have made the important observation that, because the velocity field is locally divergence-free, the singularities of functions $\alpha(\omega)$ and $k(\omega)$ - poles, zeros, and branch points - necessarily are purely imaginary (see [17] Appendix A). This characteristics of the response functions is explicitly apparent in Avellaneda and Torquato's solution of principle of the problem (4.6), written in terms of the Stokes operator's eigenmodes and relaxation times 45].

For the functions $\alpha^{\prime}(\omega)$ and $k^{\prime}(\omega)$, it was similarly shown 20 that, because 
the excess pressure field can be considered gradient-free 11 , the singularities of functions $\alpha^{\prime}(\omega)$ and $k^{\prime}(\omega)$ also are purely imaginary (see [10] Appendix C). Again, this characteristics of the response functions is explicitly apparent in a solution of principle of the problem (8) , written in terms of the Laplace operator's eigenmodes and relaxation times [20, in exactly the same manner as 45 .

The wanted functions must therefore have simple smooth behaviors on the real axis. Indeed, the DRT (distribution of relaxation times) formalism of Avellaneda and Torquato may be used to explicitly show that in the Laplace domain $(s=-i \omega>0)$ the hydrodynamic drag function $\lambda(s)=1-1 / \alpha(s)$ is always a strictly decreasing positive function on the real axis $s>0$, and the same holds true in the same manner for the corresponding thermal function $\lambda^{\prime}(s)=1-1 / \alpha^{\prime}(s)-$ see [20]. Alternatively, more recently, the strictly decreasing nature of functions $\Re \alpha(\omega)$ and $\omega \Im \alpha(\omega)$ versus real frequency has been shown in elegant manner by a variational formulation making use of the divergence-free nature (5) of the microscopic flow fields [51.

Finally, as observed by Johnson et al. [17, due to the special location of their singularities and the adjacent hypothesis of the absence of very different pore sizes, the functions $k(\omega)$ and $\alpha(\omega)$ as well as the functions $k^{\prime}(\omega)$ and $\alpha^{\prime}(\omega)$, may be seeked as the simplest ones satisfying both the frozen and relaxed limits.

To proceed, let us introduce a Stokes number constructed using Johnson's concept of dynamically connected pore size $\Lambda$, viz:

$$
S_{T}=\Lambda \sqrt{\frac{-i \omega \rho_{f}}{\eta}} .
$$

Johnson has proposed the following expression of dynamic tortuosity $\alpha(\omega)$,

$$
\alpha(\omega)=\alpha_{\infty}\left(1+\frac{8}{M S_{T}^{2}} \sqrt{1+\frac{M^{2}}{16} S_{T}^{2}}\right)
$$

where

$$
M=\frac{8 \alpha_{\infty} k_{0}}{\phi \Lambda^{2}}
$$

is a dimensionless shape factor associated to the geometry. This expression is the simplest analytical ansatz that yields the exact first two terms at high frequencies (Eq. (14)) and the exact first leading term at low frequencies (Eq. (19)), and automatically satisfies the condition on singularities. As such, and for the general reasons discussed before, it is expected to provide a reasonable description of the exact function $\alpha(\omega)$. Factors of 8 are introduced for convenience in Eqs. (25,26), so that $M=1$ for cylindrical circular pores.

Similarly, to describe the function $\alpha^{\prime}(\omega)$ let us introduce a second Stokes number corresponding to thermal effects:

$$
S_{T}^{\prime}=\Lambda^{\prime} \sqrt{\frac{-i \omega \rho_{f} \operatorname{Pr}}{\eta}} .
$$

\footnotetext{
${ }^{11}$ See footnote 7
} 
The ratio $S_{T}^{\prime} / S_{T}=\sqrt{\operatorname{Pr}} \Lambda^{\prime} / \Lambda$ is of the order of $\operatorname{Pr}^{1 / 2}$, not far from unity. Proceeding as did Johnson, Lafarge [20] proposed to write,

$$
\alpha^{\prime}(\omega)=1+\frac{8}{M^{\prime} S_{T}^{\prime 2}} \sqrt{1+\frac{M^{\prime 2}}{16} S_{T}^{\prime 2}}
$$

where

$$
M^{\prime}=\frac{8 k_{0}^{\prime}}{\phi \Lambda^{\prime 2}},
$$

is the thermal counterpart of shape factor $M\left(M^{\prime}=1\right.$ for cylindrical circular pores), giving in turn a definite model for the dynamic compressibility

$$
\beta(\omega)=\gamma-(\gamma-1) / \alpha^{\prime}(\omega) .
$$

As this model is the elaboration of Allard's original attempt to transpose Johnson's modelling to thermal effects [18, we refer to the combined modeling of functions $\alpha$ and $\beta$ Eqs. (24 301) as to Johnson-Allard's (JA).

Subsequently, Pride et al. 23, while studying oscillating viscous flow in convergent-divergent channels, found that the simple formula (25) may significantly underestimate the imaginary part of dynamic permeability $k(\omega)$ at low frequencies. To remedy this, they proposed different modified formulas. In essence these are formulas capable to account for the exact value of parameter $\alpha_{0}$, which, in such channels, may be significantly increased as compared to Johnson's. Notwithstanding, this parameter $\alpha_{0}$ is not singled out in [23. Its identification by Eq. (21) (see 9] and 20]) shows that it is constructed like the tortuosity $\alpha_{\infty}$, this time for the 'Poiseuille'-like velocity pattern. Therefore it is a measure of 'disorder' of the 'Poiseuille' flow, and as such it is increased not only by the convergent-divergent mechanism considered by Pride et al., but also, e.g., by irregularities in the distribution of solid inclusions leading to the existence of privileged flow paths. Whatever the cause of the enhanced 'Poiseuille disorder', a significant increase of factor $\alpha_{0}$ as compared to Johnson's value $\alpha_{0}=\alpha_{\infty}\left(1+\frac{M}{4}\right)$, will make it more necessary to modify Johnson's formula. Similar formal considerations hold true also, mutatis mutandis, for thermal effects and the function $\alpha^{\prime}$. Here also, a significant increase of $\alpha_{0}^{\prime}$ may result when replacing a regular distribution of solid inclusions by an irregular one (which leaves unchanged the thermal characteristic length $\Lambda^{\prime}$ ).

Now, among the different modifications proposed in 23 the first was the simplest one, capable to yield the exact first two terms at high and low frequencies, and simultaneously, to automatically fulfil the condition on singularities whatever the values of parameters $\phi, k_{0}, \alpha_{0}, \Lambda$, and $\alpha_{\alpha} 12$. Finally, this formula was expressed by Lafarge in terms of the parameter $\alpha_{0}$ and the same description was then immediately transferable to thermal effects. The corresponding

\footnotetext{
${ }^{12}$ Incidentally, this nice feature of the formula was missed in [23] and [20]: in [23] there are mistaken considerations concerning the formula, corrected in 20 but still with mistaken considerations on the singularities, repeated in [24].
} 
Pride-Lafarge's $(P L)$ model formulas are:

$$
\begin{aligned}
\alpha(\omega) & =\alpha_{\infty}\left(1+\frac{8}{M S_{T}^{2}}\left(1-q+q \sqrt{1+\frac{M^{2}}{16 q^{2}} S_{T}^{2}}\right)\right), \\
\alpha^{\prime}(\omega) & =1+\frac{8}{M^{\prime} S_{T}^{\prime 2}}\left(1-q^{\prime}+q^{\prime} \sqrt{1+\frac{M^{\prime 2}}{16 q^{\prime 2}} S_{T}^{\prime 2}}\right), \\
\beta(\omega) & =\gamma-(\gamma-1) / \alpha^{\prime}(\omega),
\end{aligned}
$$

where $M$ and $M^{\prime}$ are as before, and $q$ and $q^{\prime}$ are the new shape factors given by,

$$
q=\frac{1}{\alpha_{0}-\alpha_{\infty}} \frac{2 k_{0} \alpha_{\infty}^{2}}{\phi \Lambda^{2}} ; q^{\prime}=\frac{1}{\alpha_{0}^{\prime}-1} \frac{2 k_{0}^{\prime}}{\phi \Lambda^{\prime 2}} .
$$

These expressions reduce to $J A$ 's by setting $q=q^{\prime}=1$ and are simple transformations of the latter: they apply the simple group transformation $F\left(S_{T}\right) \rightarrow 1-q+q F\left(S_{T} / q\right)$ to the basic Johnson's square root function $F_{J}\left(S_{T}\right)=\sqrt{1+\frac{M^{2}}{16} S_{T}^{2}}$. Two successive applications of the transformation (with parameters $q$ and $p$ ) yield another same transformation (with parameter $q p$ ) which preserves both low- and high-frequency limits $F\left(S_{T}\right) \rightarrow 1+O\left(S_{T}^{2}\right)$ and $F\left(S_{T}\right) \rightarrow \frac{M}{4} S_{T}$. In this way there is some unicity in the $P L$ modification, which is not extendible in obvious very simple manner.

It must be realized that, as a side result of the strong constraints imposed by the special location of singularities and as long as the geometry is relatively simple, the $P L$ expressions constructed with one more exact term than $J A$ 's at low frequencies, will also describe in a slightly more accurate manner all of the viscous and thermal relaxation 13 . Nevertheless, it should not be hoped that it is possible to gain, by means of this description, very meaningful information on the frozen parameters $\Sigma$ and $\Sigma^{\prime}$.

If the model expressions (31-34) were exact, the comparison of their highfrequency expansions with the exact ones (14) would imply

$$
q=1-\frac{3 \alpha_{\infty} k_{0}}{\phi \Sigma} ; q^{\prime}=1-\frac{3 k_{0}^{\prime}}{\phi \Sigma^{\prime}},
$$

hence giving a fixed relation between the set of relaxed and frozen parameters. But the expressions (31)32) are not exact, and the numbers $q$ and $q^{\prime}$ in (34 35), obtained by low- or high-frequency matching of these non-exact expressions, will not be the same in general. Thus we should not hope that the high-frequency expansions obtained with $P L$ model:

$$
\begin{aligned}
& \alpha(\omega)=\alpha_{\infty}\left(1+\frac{2}{S_{T}}+\frac{8(1-q)}{M S_{T}^{2}}+O\left(\frac{1}{S_{T}^{3}}\right)\right), \\
& \beta(\omega)=1+(\gamma-1)\left(\frac{2}{S_{T}^{\prime}}+\frac{8\left(1-q^{\prime}\right)}{M^{\prime} S_{T}^{\prime 2}}-\frac{4}{S_{T}^{\prime 2}}+O\left(\frac{1}{S_{T}^{\prime 3}}\right)\right),
\end{aligned}
$$

\footnotetext{
${ }^{13}$ The incorrect statement that the $P L$ description essentially improves the low frequencies if often made in literature. In reality because of the pole in $\alpha(\omega)$ and the way $\beta(\omega)$ is related to $\alpha^{\prime}(\omega)$ - see (33), the small departures between $P L$ and $J A$ are mainly perceptible in the region of intermediate frequencies.
} 
yield anything precise for the $O(2)$ terms. The example of cylindrical circular tubes may serve to illustrate this in quantitative manner.

\subsection{The case of cylindrical pores}

For a material with cylindrical circular pores of identical radius $R$ (say for simplicity, all parallel and aligned along the direction of propagation), the two boundary value problems Eqs. (446) and (8 9) determining $\alpha(\omega)$ and $\beta(\omega)$, are easily entirely stated and solved. In effect, these are nothing but the problems considered by Zwikker and Kosten 52 in simplifying (on account of the wide separation between wavelength and tube radius) the governing equations of the full Kirchhoff's theory of sound propagation in a cylindrical circular tube 53. We may say, in this respect, that the conventional equivalent-fluid theory neglecting spatial dispersion and expressed by Eqs. (3 10), is the direct generalization to the case of arbitrary geometry, of Zwikker and Kosten's classic theory. Now, Zwikker and Kosten's result is that $\alpha(\omega), \alpha^{\prime}(\omega)$ and $\beta(\omega)$ express via Bessel functions as follows:

$$
\frac{1}{\alpha(\omega)}=1-\chi(\omega) ; \frac{1}{\alpha^{\prime}(\omega)}=1-\chi(\omega \operatorname{Pr}) ; \beta(\omega)=1+(\gamma-1) \chi(\omega \operatorname{Pr}),
$$

where $\chi(\omega)$ is the following relaxation function,

$$
\chi(\omega)=\frac{2 J_{1}\left(\left(\frac{i \omega \rho_{f}}{\eta} R^{2}\right)^{1 / 2}\right)}{\left(\frac{i \omega \rho_{f}}{\eta} R^{2}\right)^{1 / 2} J_{0}\left(\left(\frac{i \omega \rho_{f}}{\eta} R^{2}\right)^{1 / 2}\right)},
$$

(smoothly varying, in a sort 'Davidson-Cole' pattern, from relaxed value 1 at low frequencies to frozen value 0 at high frequencies). Using the known smallarguments and large-arguments series and asymptotic expansions of Bessel functions (or Kelvin functions), it is simple to derive the following low-frequency and high-frequency exact behaviors:

Low frequencies:

$$
\alpha(\omega)=\frac{8 \eta}{-i \omega \rho_{f} R^{2}}+\frac{4}{3}+O(-i \omega) \quad ; \quad \alpha^{\prime}(\omega)=\frac{8 \eta}{-i \omega \rho_{f} \operatorname{Pr} R^{2}}+\frac{4}{3}+O(-i \omega)
$$

High frequencies:

$$
\begin{aligned}
\alpha(\omega) & =1+\frac{2}{R}\left(\frac{\eta}{-i \omega \rho_{f}}\right)^{1 / 2}+\frac{3}{R^{2}}\left(\frac{\eta}{-i \omega \rho_{f}}\right)+O\left(\frac{1}{-i \omega}\right)^{3 / 2} ; \\
\alpha^{\prime}(\omega) & =1+\frac{2}{R}\left(\frac{\eta}{-i \omega \rho_{f} \operatorname{Pr}}\right)^{1 / 2}+\frac{3}{R^{2}}\left(\frac{\eta}{-i \omega \rho_{f} \operatorname{Pr}}\right)+O\left(\frac{1}{-i \omega}\right)^{3 / 2}
\end{aligned}
$$

Then comparing these results with the general low- and high-frequency expansions Eqs. (19) and (14), the following parameters values are easily obtained:

$$
k_{0}=k_{0}^{\prime}=\phi R^{2} / 8 ; \alpha_{0}=\alpha_{0}^{\prime}=4 / 3 ; \Sigma=\Sigma^{\prime}=R^{2} ; \Lambda=\Lambda^{\prime}=R ; \alpha_{\infty}=1 .
$$


From Eqs. (30) and (34), the circular-tube shape factors $M, q$ and $M^{\prime}, q^{\prime}$ are identified as:

$$
M=M^{\prime}=1 ; q=q^{\prime}=3 / 4,
$$

whereas for the modified $P L$ model (35) the latter are:

$$
q=q^{\prime}=5 / 8
$$

Let us now examine how far the models are consistent with the limits (40,41) and collection of parameters (42).

When Johnson's values $q=q^{\prime}=1$ are used in the model expressions 31 32), no $S_{T}^{2}=O(-1 / i \omega)$ terms appear in the high-frequency limit (14): the characteristic surfaces $\Sigma$ and $\Sigma^{\prime}$ predicted by model $J A$ are given infinite values whatever the geometry (this can be seen also on Eqs. (35)). Simultaneously, in the low-frequency limit, the relaxed parameters $\alpha_{0}, \alpha_{0}^{\prime}$ in (19) are given as $\alpha_{0}=\alpha_{\infty}\left(1+\frac{M}{4}\right), \alpha_{0}^{\prime}=\left(1+\frac{M^{\prime}}{4}\right)$. Consider specifically the increments $\alpha_{0}-\alpha_{\infty}$ and $\alpha_{0}^{\prime}-1$, i.e. the differences $[\alpha]_{\text {relaxed }}-[\alpha]_{\text {frozen }}$ produced by the viscous and thermal relaxation processes. For the present case of cylindrical circular tubes, it follows that the relative error $\left[()_{\text {model }}-()_{\text {exact }}\right] /()_{\text {exact }}$ made by the model $J A$ on these increments, is a $(1 / 4-1 / 3) /(1 / 3)=-25 \%$ error.

When the $P L q$ and $q^{\prime}$ values (43) (i.e. (34)) are used, the latter increments are exactly described, but a $50 \%$ overestimation still exists for the characteristic surfaces: putting the values (43) in (35) yields $\Sigma=\Sigma^{\prime 2} / 2$.

When the modified $P L q$ and $q^{\prime}$ values (44) (i.e. (35)) are used, the characteristic surfaces are exactly described but there remain now a $(8 /(4 \times 5)-1 / 3) /(1 / 3)$ $=+20 \%$ error on the viscous and thermal increments.

Finally, when the values of $q$ and $q^{\prime}$ are taken as the arithmetic mean of the $P L$ and modified $P L$ ones, simultaneous but reduced errors are made: the characteristic surfaces are given with $20 \%$ overestimation (instead of $50 \%$ with $P L$ ), while the viscous and thermal increments are given with $9 \%$ error (instead of $20 \%$ with modified $P L)$.

In general, the $J A^{\prime}$ and $P L$ ' models unsatisfactory account of parameters $\Sigma$ and $\Sigma^{\prime}$, will be at the origin of some errors in the description of the propagation of transients at relatively short times or short distances, whereas the modified $P L '$ model description unsatisfactory account of parameters $\alpha_{0}$ and $\alpha_{0}^{\prime}$, will be at the origin of errors in the description of transients at longer times and distances. To study this we compare in what follows different exact (fully exact or asymptotically exact) and modelled time Green's functions for the case of cylindrical circular tubes.

Let us first define the Green's functions and write the exact asymptotic results that have been described in introduction and are more detailed in Appendix. 


\section{A simple definition of a Green's function; scaled form}

\subsection{Definition and scaled form}

A general method of defining and calculating a Green's function in an infinite medium is by means of the effective frequency-dependent wavenumber $k$ in this medium. Let us define our Green's function $G(x, t)$ (or impulse response) as a propagated Dirac delta impulsive signal $\delta(t)$ imposed at $x=0$, or more precisely, as the inverse Fourier transform of the propagation transfer function $G_{x}(\omega)=\exp (i k x)$ :

$$
G(x, t)=\int_{-\infty}^{\infty} \frac{d \omega}{2 \pi} \exp [-i \omega t+i k x] .
$$

Setting to zero the viscosity and thermal conduction coefficients, no frequency dispersion arises. The wavenumber writes $k=\omega / c$ where $c$, defined by the first Eq. (A-7), is the frozen speed of sound $c=c_{f} / \sqrt{\alpha_{\infty}}$. The Green's function (45) coincides with a Dirac delta propagated at this velocity $c: G(x, t)=\delta(t-x / c)$.

Setting to nonzero values the viscosity and thermal conduction coefficients the medium wavenumber $k$ writes,

$$
k=\frac{\omega}{c} \sqrt{\frac{\alpha(\omega) \beta(\omega)}{\alpha_{\infty}}}=\frac{\omega}{c}\left[1+h\left(S_{T}\right)\right],
$$

making apparent a complex function $h\left(S_{T}\right)$ that describes frequency dispersion induced by the viscous and thermal relaxation processes. The Green's function (45) writes,

$$
G(x, t)=\int_{-\infty}^{\infty} \frac{d \omega}{2 \pi} \exp \left[-i \omega(t-x / c)+i \frac{\omega}{c} h\left(S_{T}\right) x\right] .
$$

This Green's function $g_{x}(t-x / c)=G(x, t)$ now extends and flattens when observed at positions $x$ more and more remote from the origin $x=0$. Explicit asymptotic expressions of the Green's function defined in this manner will now be obtained by considering high-frequency asymptotic expansions of the wavenumber $k$ (meaning high-frequency asymptotic expansions of the function $h)$.

It will also be convenient to express these functions in scaled form, as functions of a dimensionless delay time $\tau$ and a dimensionless position variable $\xi$. To take but one example, before being Fourier-transformed, the Green's function $G_{x}(\omega)=\exp (i k x)$ where $k$ is - say - given by the model $P L$, may be viewed as a function of:

( $i$ ) one dimensionless frequency variable, e.g. $\Omega=\omega \Theta$ where $\Theta$ is the characteristic viscous relaxation time given by,

$$
\Theta=\frac{\Lambda^{2} \rho_{f}}{\eta}
$$


(hence $S_{T}^{2}=-i \Omega$ ),

(ii) one dimensionless position variable, e.g.

$$
\xi=\frac{x}{c \Theta},
$$

(hence $\xi^{-1 / 2}=\Lambda \sqrt{\rho_{f} c /(x \eta)}$ can be regarded as a time domain Stokes number, when replacing $-i \omega$ by $c / x$ in Eq. (24)).

(iii) a number of dimensionless parameters characteristic of the form of the porous space but not of its absolute dimensions $\left(\alpha_{\infty}, M, q, M^{\prime}, q^{\prime}\right.$, and $\left.\Lambda^{\prime} / \Lambda\right)$, and,

(iv) two dimensionless parameters characteristic of the fluid ( $\gamma$ and $\operatorname{Pr}$ ).

Suppose that the parameters (iii) and (iv) of both the medium and the fluid are held constant. Function $G_{x}(\omega)=\exp (i k x)$ then reduces to a function of $\Omega$ which is parameterized by $\xi$. There follows that the shape of the corresponding time-domain function will depend, in scaled form, on $\xi$ only, provided the time is counted in a dimensionless manner, e.g. for the time elapsed after the first arrival of the signal,

$$
\tau=\left(t-\frac{x}{c}\right) / \Theta .
$$

But function $G(x, t)$ has the dimension of the inverse of time. Therefore,

$$
\Theta G(x, t)=F_{s}(\xi, \tau),
$$

with $F_{s}$ a scale-invariant function that depends on the form of the pore space but not on its absolute dimensions:

$$
F_{s}(\xi, \tau)=\int_{-\infty}^{\infty} \frac{d \Omega}{2 \pi} \exp [-i \Omega \tau+i \Omega \xi h(\sqrt{-i \Omega})] .
$$

Evidently, function $F_{s}$ will depend on the description of wavenumber or function $h\left(S_{T}\right)$ : model $J A$, model $P L$, only some high-frequency terms retained, or complete exact Zwikker and Kosten's.

\subsection{Asymptotic expansions}

Two successive closed-form analytical exact asymptotic expressions can be derived for the frozen limit, using the series expansion of the wavenumber for the case where the function $h\left(S_{T}\right)$ is represented by its first, and second order terms on $S_{T}^{-1}$. In the first case, using (Eqs. (14) and (46)), we write:

$$
h=\frac{2 n_{1}}{\sqrt{-i \Omega}}+o(1)
$$

where,

$$
2 n_{1}=1+(\gamma-1) \frac{\Lambda}{\Lambda^{\prime} \sqrt{\mathrm{Pr}}}
$$

and obtain,

$$
\begin{aligned}
& \Theta G_{o(1)}(x, t)=0 \text { for } \tau<0, \\
& \Theta G_{o(1)}(x, t)=F_{s 1}(\xi, \tau) \text { for } \tau>0,
\end{aligned}
$$


where,

$$
F_{s 1}(\xi, \tau)=\frac{1}{\sqrt{\pi}} \frac{n_{1} \xi}{\tau^{3 / 2}} \exp \left[-n_{1}^{2} \frac{\xi^{2}}{\tau}\right] .
$$

In the second case we write

$$
h=\frac{2 n_{1}}{\sqrt{-i \Omega}}+\frac{n_{2}}{-i \Omega}+o(2),
$$

where,

$$
2 n_{2}=\frac{3 \Lambda^{2}}{\Sigma}-1+\frac{2(\gamma-1) \Lambda}{\Lambda^{\prime} \sqrt{\operatorname{Pr}}}+\frac{(\gamma-1) \Lambda^{2}}{\Lambda^{\prime 2} \operatorname{Pr}}\left(\frac{3 \Lambda^{\prime 2}}{\Sigma^{\prime}}-3-\gamma\right),
$$

and obtain,

$$
\begin{aligned}
\Theta G_{o(2)}(x, t) & =0 \text { for } \tau<0, \\
\Theta G_{o(2)}(x, t) & =F_{s 1}(\xi, \tau) \exp \left(-n_{2} \xi\right) \text { for } \tau>0 .
\end{aligned}
$$

This yields an attenuation without distortion, corresponding to the asymptotic results indicated in Eqs. (12). This analytical form of the Green's function, exhibiting a superimposed exponential decay, is also a known result of musical acoustics 39 .

Concerning the relaxed limit, the purely diffusive Green's function $G_{d i f f}$ can be obtained by assimilating $\beta(\omega)$ with $\gamma$ and $\alpha(\omega)$ with its first leading term $\eta \phi /\left(-i \omega \rho_{f} k_{0}\right)$ in Eq. (19). In this relaxed d.c. approximation, the wavenumber expands as:

$$
k=k_{\text {diff }}=\frac{1}{c}\left(\frac{8 \gamma}{M \Theta}\right)^{1 / 2} \sqrt{i \omega} .
$$

(we have used the relation (26)). The corresponding analytical form of the Green's function is deduced from the known result given by Ref. [57]:

$$
\begin{aligned}
& \Theta G_{\text {diff }}(x, t)=0 \text { for } \tau^{\prime}<0 \\
& \Theta G_{\text {diff }}(x, t)=F_{s, \text { diff }}\left(\xi, \tau^{\prime}\right) \text { for } \tau^{\prime}>0,
\end{aligned}
$$

where the notation $\tau^{\prime}=t / \Theta$ is used to avoid confusion with Eq. (50):

$$
F_{s, d i f f}\left(\xi, \tau^{\prime}\right)=\frac{1}{\sqrt{\pi}} \sqrt{\frac{2 \gamma}{M}} \frac{\xi}{\tau^{\prime 3 / 2}} \exp \left[-\frac{2 \gamma}{M} \frac{\xi^{2}}{\tau^{\prime}}\right] .
$$

\section{FFT computation of Green's functions (full- frequency expressions)}

Green's functions $G(x, t)$ computed numerically by inverse FFT (fast Fourier transform) of the transfer function $\exp (i k x)$, can always be obtained as soon as exact or approximate full-frequency expressions of the wavenumber $k$ in Eq. (46) are known. These FFT computations are to be carefully done, however, as it is detailed below. In Appendix, the different results, either analytical or numerical, corresponding to the asymptotic Green's functions are summarized 
and compared with the exact results of the FFT.

For the case of arbitrary porous media, approximate models such as $J A$ or $P L$ may be used for $k(\omega)$, leading to the computation, by FFT, of approximate Green's functions $G(x, t)$. For the case of simple workable geometries (e.g., cylindrical circular aligned pores of identical radius) exact expressions are available, leading to the computation, by FFT, of exact Green's functions $G(x, t)$. For later use, it will be convenient to distinguish and denote respectively by:

$G$ the exact (Zwikker and Kosten) Green's function;

$G_{J A}$ the full $J A$ Green's function $\left(q=q^{\prime}=1\right)$;

$G_{P L}$ the full $P L$ Green's function $\left(q=q^{\prime}=3 / 4\right)$;

$G_{m P L}$ the modified full $P L$ Green's function (defined by $P L$ 's expressions with $q=q^{\prime}=5 / 8$;

$G_{o(1)}$ the above asymptotic Green's function obtained by retaining in the wavenumber the zero and first frozen terms;

$G_{o(2)}$ the above asymptotic Green's function obtained by retaining in the wavenumber the zero, first and second frozen terms.

\subsection{The use of the asymptotic diffusive Green's function in the FFT computation}

An important point is that the analytical diffusion solution $G_{\text {diff }}$ given by Eq. (61) is used in our FFT computations to improve the accuracy of calculations. Indeed, the numerical computation of the full-frequency exact and model Green's functions must be done by FFT with some precautions: because of the importance of the higher frequencies on the response shape, subtracting the diffusive (low frequencies) approximation largely improves the results. Thus, instead of calculating directly the inverse Fourier transform $G(x, t)$ of the function $\exp (i k x)$, we first compute the inverse Fourier transform $G^{\prime}(x, t)$ of the function $\exp (i k x)-\exp \left(i k_{d i f f} x\right)$ : The latter difference being zero at zero frequency, and increasing smoothly then decreasing rapidly toward zero at high frequency, the function $G_{x}(\omega)$ is naturally windowed in the frequency domain. And then we use the relation $G(x, t)=G^{\prime}(x, t)+G_{d i f f}(x, t)$, with $G_{d i f f}(x, t)$ as given by the Eqs. (61,62) above (see Ref. [54). The validity of the FFT computation has been checked for first order asymptotic expression $G_{o(1)}$, with an accuracy better than $1 \%$.

\subsection{Computed results: time responses}

For the computation, the values of the parameters have been chosen close to those of material M1 in Ref. 25. The porosity is $\phi=0.82$, the flow resistivity $\sigma=196000 \mathrm{Nm}^{-4} s$, the permeability $k_{0}=\eta / \sigma=9.22510^{-11} \mathrm{~m}^{2}$, corresponding to a radius $R=310^{-5} \mathrm{~m}$. The temperature is $20^{\circ} \mathrm{C}$, and the Prandtl number $\operatorname{Pr}=0.71$. The characteristic viscous relaxation time is $\Theta=6 \cdot 10^{-5} \mathrm{~s}$.

The chosen length is $5.3 \mathrm{~mm}$ (the dimensionless length is $\xi=0.3152$ ). Fig. 1 shows the results for the dimensionless Green's function, obtained by FFT. The Pride-Lafarge description is compared with the exact Zwikker and Kosten formula. As expected, the full $P L$ description is very satisfactory for short times (high frequencies) and long times (low frequencies). For very long times, both descriptions reach the diffusive (analytical) limit, i.e. the Poiseuille behavior is reached. 


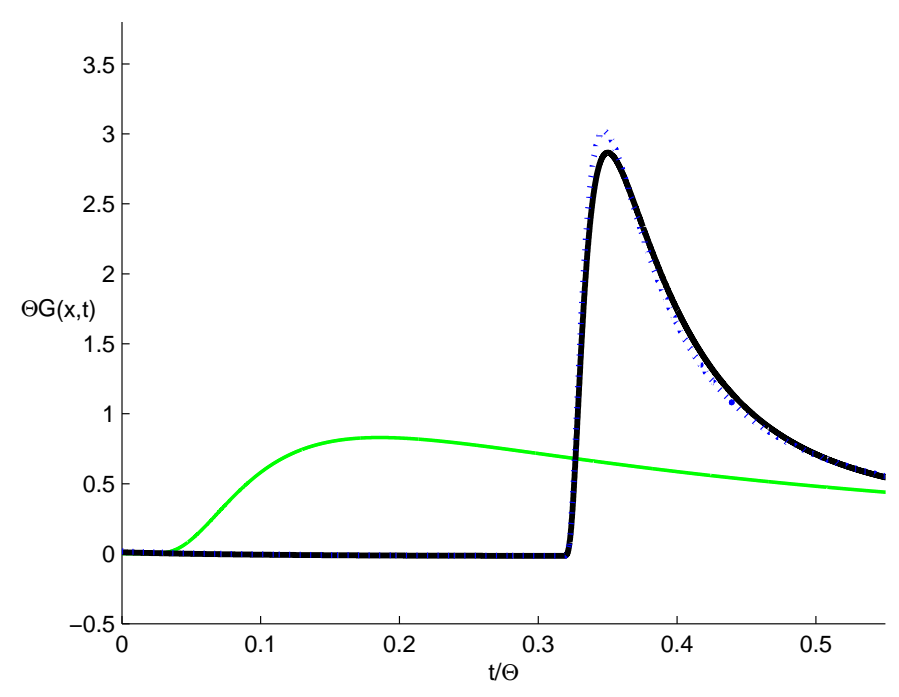

Figure 1: Green's function $\Theta G(x, t)$ with respect to time $t / \Theta$ (both are dimensionless). The characteristic viscous relaxation time $\Theta$ is defined by Eq. (48). (Black) solid line: Zwikker and Kosten formulae (Eqs. (38) and (39)). (Blue) dotted line: Pride-Lafarge description (Eqs. (31) to (34)). (Green) thin solid line: diffusive limit (Eqs. (61) and (62)). For very long times, the diffusive limit is reached.

In order to emphasize these results, Fig. 2 shows a zoom of the previous figure, and other approximations have been added. The modified $P L$ Green's function, denoted $G_{m P L}$, can be compared to the $P L$ Green's function. As expected, it is more accurate for short time (during the signal rise) than the original $P L$ function, but it is less accurate for long times, because the choice of the parameters $q$ and $q^{\prime}$ has been done from the frozen limit instead of the relaxed limit. Otherwise the $J A$ model yields less accurate results than both $P L$ models. The asymptotic Green's function $G_{O(2)}$ which uses the same number of parameters as $J A$ performs quite well. On Fig. 2 it is observed that the models $P L$ and $m P L$ are in error mainly in the region of the maximum; one elementary means to improve the description of the bump shape of the response would be to take the direct mean of the two models Green's functions. The response modelled in this manne1 14 would be very close to the exact one. Additional results given later on (Fig.4) show however that this is a favourable situation related to the value $\xi \simeq 0.3$ and that no very significant improvement of model $P L$ is to be expected in this manner for other $\xi$ values. 


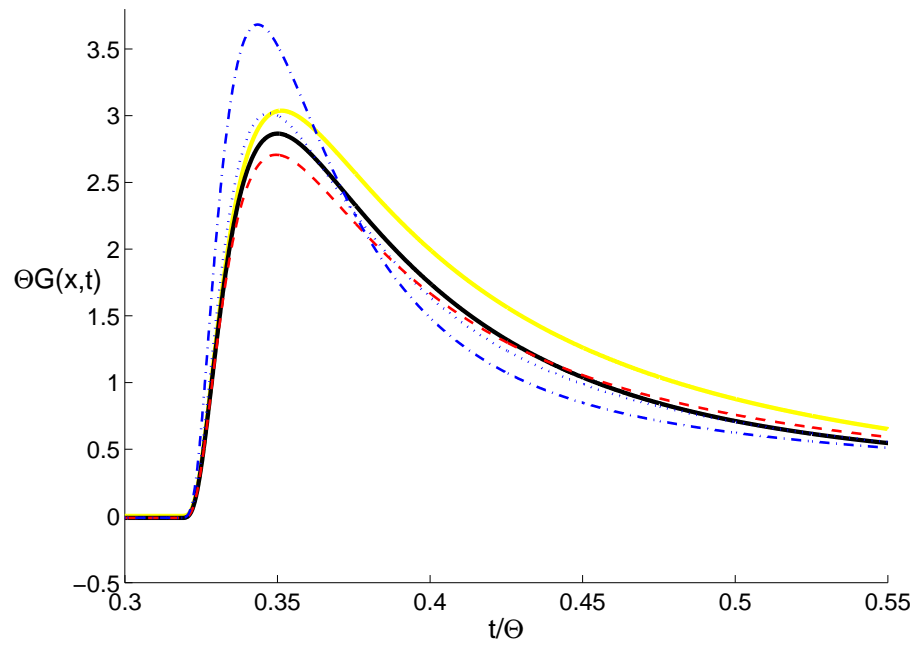

Figure 2: Green's function $\Theta G(x, t)$ with respect to time $t / \Theta$ (zoom of figure 1). (Black), solid line: Zwikker and Kosten formula. (Blue), dotted line: $P L$ formula. (Red), dashed line: $P L$ modified formula. (Blue), mixed line: $J A$ formula. (Yellow), solid, pale line: frozen $o(2)$ 's Green's function $G_{o(2)}$ - see Appendix, Eqs. (56- 59).

\subsection{Computed results: Maximum values of the time re- sponse}

The previous results are concerned with a fixed value of the parameter $\xi$, i.e. a fixed value of the thickness of the material layer. In order to compare the different descriptions for several values of $\xi$, we chose to compare the maximum values of the time responses (there is a unique maximum). For the asymptotic (frozen) expressions at the first orders, $F_{s 1}$ and $F_{s 2}$, the maximum values are given by an analytical expression, obtained from Eq. (56):

$$
\begin{aligned}
\operatorname{Max}\left(F_{s 1}\right) & =\frac{1}{\sqrt{\pi}}\left[\frac{3}{2}\right]^{3 / 2} e^{-(3 / 2)} \frac{1}{n_{1}^{2} \xi^{2}}=\frac{0.2312}{n_{1}^{2} \xi^{2}} ; \\
\operatorname{Max}\left(F_{s 2}\right) & =\operatorname{Max}\left(F_{s 1}\right) \exp \left(-n_{2} \xi\right) .
\end{aligned}
$$

For both orders the time $\tau_{\max }=2 n_{1}^{2} \xi^{2} / 3$ of the maximum is the same (this illustrates the attenuation without distortion effect brought by the $O(2)$ terms). Fig. 3 shows the result for the exact Green's function $G$ and the two $P L$ models, $G_{P L}$ and $G_{m P L}$, as well as the diffusive function $G_{d i f f}$. It shows the natural logarithm of the maximum value of $F_{s}$ with respect to the dimensionless space variable $\xi$. The two $P L$ models seem to be very good; however better insight is found by subtracting the result corresponding to the exact Green's function, as shown in Fig. 4. As expected, the $P L$ description is very good for long distances $\xi$, while the modified $P L$ description is better for small distances $\xi$. Otherwise

\footnotetext{
${ }^{14}$ Or, with almost undistinguishable results, with model $P L$ calculated with values of $q$ and $q^{\prime}$ equal to the mean of their original and modified $P L$ values.
} 


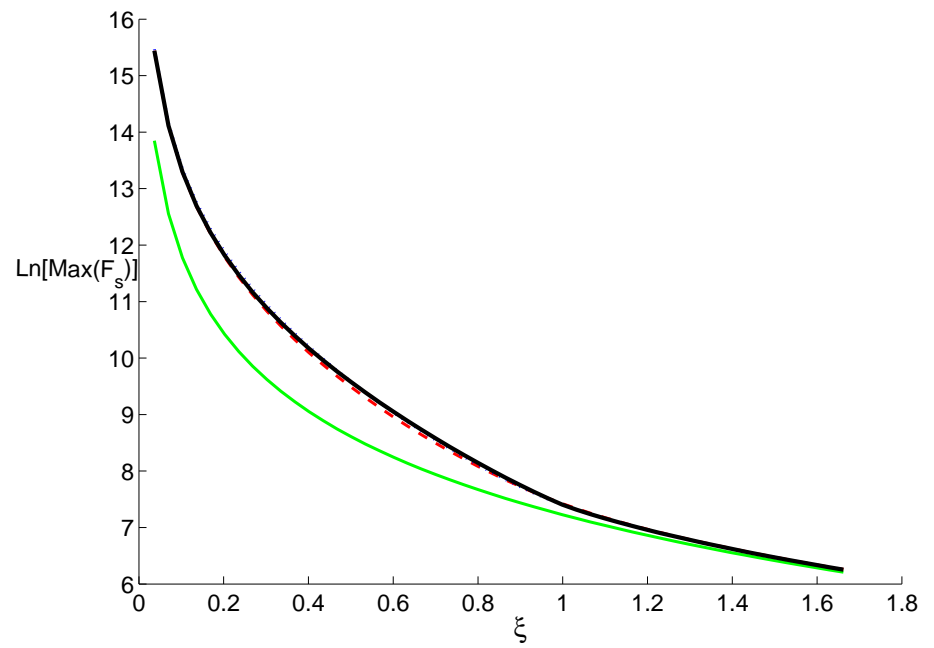

Figure 3: Natural logarithm of the Green's function maximum. (Black) solid thick line: exact Green's function $G$. (Red) dashed line: modified Green's function $G_{m P L}$. (Green) solid thin line: diffusive Green's function $G_{d i f f}$.(Blue) dotted line: $P L$ Green function $G_{P L}$ - at this scale this curve cannot be distinguished from the exact result, see Fig. 团 for details.

both are better than the $J A$ description. The transition range values of the distance $\xi$ is approximately between 0.036 and 1.7 , corresponding to a range for the time domain Stokes number $5.3 \geq \xi^{-1 / 2} \geq 0.75$. This range is similar to that accepted for the frequency domain Stokes number defined by Eq. (24) (see e.g. Ref. [56]).

\section{Conclusion}

A simple analytic formula, Eq.(1), can be used to compute the $o(2)$ asymptotic Green's function in a rigid-framed porous medium. It differs from the $o(1)$ asymptotic Green's function, by an exponential factor $\exp (-x / \mathcal{L})$ which describes an attenuation-without-distortion effect.

We have so far no rigorous statements concerning the geometrical surfaces $\Sigma$ and $\Sigma^{\prime}$ that determine the $O(2)$ terms in the high-frequency limits (14) and then also determine the characteristic decay length $\mathcal{L}$ Eq. (2). Nevertheless these parameters are known for some geometries, such as cylindrical pores.

By specializing to this particular geometry, a contrasted situation has been highlighted: while the models fail to give the parameters $\Sigma, \Sigma^{\prime}$, and thus, a correct description of the $o(2)$ attenuation-without-distortion effect, they are nevertheless capable to give - especially the model $P L-$ a relatively precise description of the complete Green's functions. Indeed, it is only at very short distances that the asymptotic $o(2)$ Green's function is close to the complete Green's function: its imperfect representation by the models is of no very significant consequence.

In connection with this, we note that, when the normalized distance $\xi$ de- 


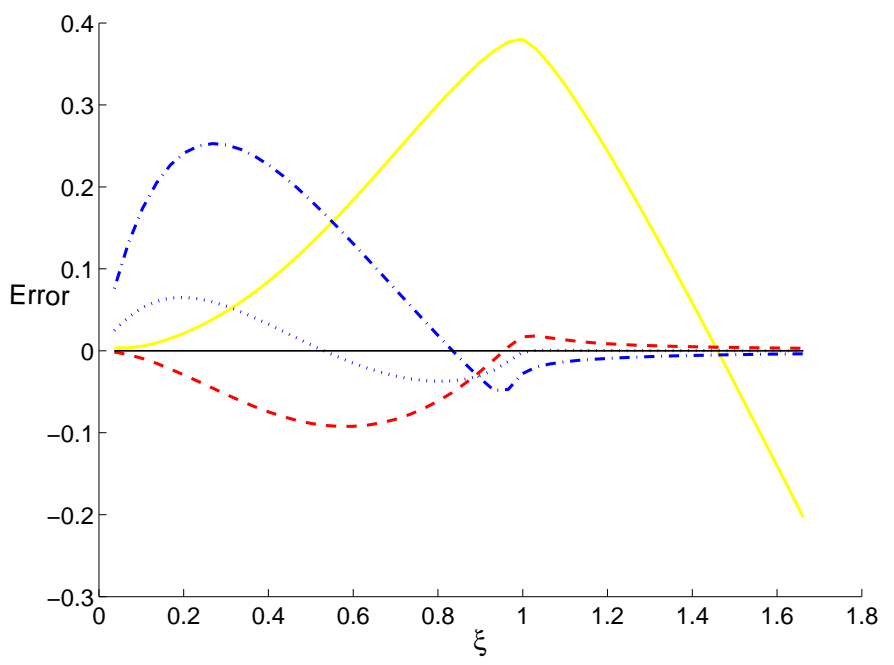

Figure 4: Error on the natural logarithm of the Green's function maximum. The different solutions are compared to the exact one, the error being the difference between the corresponding value and the exact value. (Blue) dotted line: $G_{P L}$. (Red) dashed line: modified $P L G_{m P L}$. (Blue) mixed line: $G_{J A}$. (Yellow) solid line: frozen $o(2)$ 's Green's function $G_{o(2)}$ - see Appendix, Eqs. (56- [59).

creases, the maximum error of $P L$ 's model occurs around $\xi=0.2$, precisely when the $o(2)$ Green's function eventually starts to be valid (see Fig. 4). This suggests that the small remaining errors of $P L$ 's model illustrated in Fig. 4 are mainly due to the misrepresentation of parameters $\Sigma, \Sigma^{\prime}$, and that the model would become almost exact if modified to properly account for the latter parameters. The question of the modification to be done remains open.

A problem of major interest is the use of the present investigation for the inverse problem, i.e. the determination of the parameters of a given material. Regarding this, we have illustrated one simple fact: the description of the time domain Green's functions is much more precise using the full model expressions than using the asymptotic expressions, as often done in practice.

This suggests that there is also an important potential of improvement of the inverse methods of characterization based on recording transmitted and reflected pulses on different thicknesses of a material, provided the full expressions are exploited in the analysis - we emphasize, in this respect, the importance of the substraction of the diffusive solution when computing the inverse FFT. 


\section{APPENDIX: Asymptotic expressions (frozen limit)}

\section{Asymptotic $o(1)$ 's and $o(2)$ 's expressions of the Green's function}

Recall that, since we assume a smooth pore-surface interface, in the frozen limit the product $\alpha(\omega) \beta(\omega) / \alpha_{\infty}$ in Eq. (46) expands in successive integral powers of $S_{T}^{-1}$ (see footnote 9). This has been done with dimensionless variables in section 3.2 . The two coefficients $n_{1}$ and $n_{2}$ have been obtained using the a priori expansions of the functions $\alpha(\omega)$ and $\alpha^{\prime}(\omega)$ (Eqs. (14)). For the case of cylindrical circular pores, the result for $n_{2}$ has been given by Keefe [56], using the Zwikker and Kosten solution:

$$
n_{2}=1+\frac{\gamma-1}{\sqrt{\operatorname{Pr}}}-\frac{\gamma(\gamma-1)}{2 \operatorname{Pr}}
$$

(Eq (A-1) also follows by putting the values (42) in Eq. (58)). For the general case, $n_{2}$ requires the missing frozen $O(2)$ information $\Sigma$ and $\Sigma^{\prime}$. It will not be given by the asymptotic expansion, Eqs. (36) and (37), with either $J A$ or $P L$ values of $q$ and $q^{\prime}$. As explained in section 2.2 , in the framework of the $P L$ model, it is not possible to have a good estimation of the second order term, resulting in an expression for the coefficient $n_{2}$ which differs from Eq. (58):

$$
2 n_{2}=\frac{8(1-q)}{M}-1+\frac{2(\gamma-1) \Lambda}{\Lambda^{\prime} \sqrt{\operatorname{Pr}}}+\frac{(\gamma-1) \Lambda^{2}}{\Lambda^{\prime 2} \operatorname{Pr}}\left(\frac{8\left(1-q^{\prime}\right)}{M^{\prime}}-3-\gamma\right) .
$$

The case of cylindrical circular pores allows checking this. For this case, the latter equation becomes:

$$
n_{2}=\frac{1}{2}+\frac{\gamma-1}{\sqrt{\mathrm{Pr}}}-\frac{\gamma^{2}-1}{2 \mathrm{Pr}}
$$

This expression differs from Eq. A-1 . For standard conditions in air, the exact result for $n_{2}$ is 1.08 , while the approximated one is 0.29 , i.e. more than three times smaller. Notice that $J A$ model would give a negative estimate of -1.27 for it. These important discrepancies mean that the models $J A$ and even $P L$ will not be able to describe the exact $o(2)$ attenuation effect.

\section{Asymptotic o(2) wave equation}

An alternative to the closed-form Green's function obtained for this same $o(2)$ asymptotic frozen limit can be obtained using the 1D wave equation that follows from Eqs. (1), i.e. the following Helmholtz equation:

$$
\frac{d^{2} p}{d x^{2}}+\omega^{2} \frac{\rho_{f} \alpha(\omega) \beta(\omega)}{K_{f}} p=0
$$


Using the high-frequencies asymptotic limits (14) we get,

$$
\begin{aligned}
\alpha(\omega) \beta(\omega) / \alpha_{\infty}= & 1+\frac{2}{S_{T}}+(\gamma-1) \frac{2}{S_{T}^{\prime}}+\frac{3 \Lambda^{2}}{\Sigma S_{T}^{2}}+ \\
& (\gamma-1)\left(\frac{3 \Lambda^{\prime 2}}{\Sigma^{\prime} S_{T}^{\prime 2}}-\frac{4}{S_{T}^{\prime 2}}+\frac{4}{S_{T} S_{T}^{\prime}}\right)+o(2),
\end{aligned}
$$

In the time domain, the corresponding asymptotic wave equation is written as follows 25]:

$$
\frac{\partial^{2} p(x, t)}{\partial x^{2}}-\mathcal{A} \frac{\partial^{2} p(x, t)}{\partial t^{2}}-\mathcal{B} \int_{0}^{t} \frac{\partial^{2} p\left(x, t^{\prime}\right) / \partial t^{\prime 2}}{\sqrt{t-t^{\prime}}} d t^{\prime}-\mathcal{C} \frac{\partial p(x, t)}{\partial t}=0 .
$$

Comparison between (A-4) $(\mathrm{A}-5)$ and $(\mathrm{A}-6)$ shows that the coefficients are given by:

$$
\begin{aligned}
\mathcal{A} & =\frac{1}{c^{2}}=\frac{\rho_{f} \alpha_{\infty}}{K_{f}}, \quad \mathcal{B}=4 n_{1} \sqrt{\frac{1}{\pi}} \frac{1}{\Lambda c^{2}} \sqrt{\frac{\eta}{\rho_{f}}} \\
\mathcal{C} & =\frac{1}{c^{2}} \frac{\eta}{\rho_{f}} \frac{1}{\Lambda^{2}} m \text { with } \\
m & =\frac{3 \Lambda^{2}}{\Sigma}+(\gamma-1)\left[\frac{4 \Lambda}{\Lambda^{\prime} \sqrt{\mathrm{Pr}}}+\left(-4+\frac{3 \Lambda^{\prime 2}}{\Sigma^{\prime}}\right) \frac{\Lambda^{2}}{\Lambda^{\prime 2} \mathrm{Pr}}\right] .
\end{aligned}
$$

The relationship between the coefficients $m$ and $n_{1}$ and $n_{2}$ is:

$$
m=2 n_{2}+4 n_{1}^{2}
$$

Notice that by using $P L$ model one would arrive in the asymptotic highfrequency limit to the same asymptotic wave Eq. (A-6) but with the following erroneous value of the index $m$ :

$$
m=\frac{8(1-q)}{M}+(\gamma-1)\left[\frac{4 \Lambda}{\Lambda^{\prime} \sqrt{\mathrm{Pr}}}+\left(-4+\frac{8\left(1-q^{\prime}\right)}{M^{\prime}}\right) \frac{\Lambda^{2}}{\Lambda^{\prime 2} \mathrm{Pr}}\right] .
$$

In particular, using $J A$ model, two important terms disappear as for this case one sets $q=q^{\prime}=1$. Using this expression for the index $m$ in (A-8) corresponds to using the equations (14-16) of Fellah et al. 25], who computed the Green's function for an infinite medium described by the above wave equation (A-6), by using the Laplace transform method 15 .

\section{Comparison of the asymptotic expressions}

The FFT computations of the exact Green's function can be compared with the following expressions:

$$
G_{o(1)} \text { the (frozen) } o(1) \text { Green's function; }
$$

\footnotetext{
${ }^{15}$ Notice that in Ref. 25] there was a mistake of a factor 2 in the term under the root in Eqs. (31) and (32), without influence on further equations. Moreover the last term $-4 / S_{T}^{\prime 2}$ in Eq. (37) was omitted, resulting in a total coefficient of the term in $S_{T}^{\prime-2}$ in Eq (37) equal to +2 . Here, consistent with Eq. (37), an additional term has been included in the bracket in Eq. A-11).
} 
$G_{o(2)}$ the (frozen) $o(2)$ Green's function (Eq. (59) with $q=q^{\prime}=5 / 8$ in Eq. $(\mathrm{A}-2)$; (A-2) ;

$G_{J A o(2)}$ the $J A o(2)$ Green's function (Eq. (59) with $q=q^{\prime}=1$ in Eq.

$G_{P L o(2)}$ the $P L o(2)$ Green's function (Eq. (59) with $q=q^{\prime}=3 / 4$ in Eq. (A-2)).

Finally, the FFT computations can be compared to the solution of the asymptotic $o(2)$ wave equation (A-6), for both cases $q=q^{\prime}=5 / 8$ and $q=$ $q^{\prime}=3 / 4$ (we again choose to compute these solutions using FFT, with Eqs. (46) and (A-5), without expansion of Eq. (46) ):

$G_{W E o(2)}$ the (frozen) $o(2)$ Green's function $\left(q=q^{\prime}=5 / 8\right)$;

$G_{W E P L o(2)}$ the $P L o(2)$ Green's function $\left(q=q^{\prime}=3 / 4\right)$.

These solutions being $o(2)$ are expected to be very close to the corresponding solutions obtained using the asymptotic $o(2)$ wavenumber.

Results are plotted on Fig. 5. Notice that $G_{o(2)}$, the (frozen) $o(2)$ Green's function, which is a very simple analytical expression, is the best approximation and leads to interesting results, except at long times.

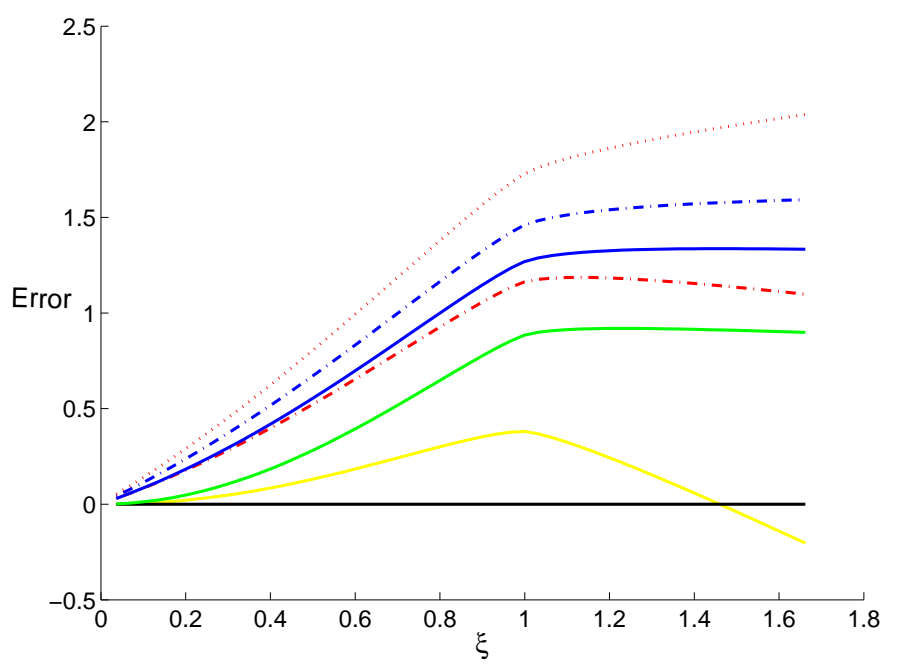

Figure 5: Error on the natural logarithm of the Green's function maximum. The different asymptotic solutions are compared to the exact one, the error being the difference between the corresponding value and the exact value. From the largest to the smaller error, the following curves represent respectively: $G_{J A o(2)}$; $G_{o(1)} ; G_{W E P L o(2)} ; G_{P L o(2)} ; G_{W E o(2)} ; G_{o(2)}$, the latter being also shown in Fig. 4 .

The $P L o(2)$ description is slightly better than the first order of the frozen asymptotic $G_{o(1)}$, but as expected, it is much comparable to the latter, as it severely underestimate the decay length $\mathcal{L}$. Otherwise, for short distances $\xi$, the second order of the asymptotic wave equation solution $G_{W E o(2)}$ exhibits the expected convergence to the results of the solution $G_{o(2)}$ based on wavenumber expansion at second order. This convergence is lost for the comparable $P L$ model estimates $G_{W E P L o(2)}$ and $G_{P L o(2)}$, as a result of using the faulty 
$P L$ coefficients $\left(q=q^{\prime}=3 / 4\right)$. For longer distances, the frozen asymptotic $W E$ solution $G_{W E o(2)}$ appears to be less accurate than the frozen asymptotic wavenumber solution $G_{o(2)}$ : it is not easy to have an interpretation for this result. The second order solution of the wave equation, as presented in Ref. 25] differs (by definition) by the third order, with the solution based on wavenumber expansion at second order, the latter being simpler to use in practice. 16 .

\section{Acknowledgments}

We wish to thank Bruno Lombard for fruitful discussions.

\section{References}

[1] A. Bensoussan, J.L. Lions, G.C. Papanicolaou, Asymptotic analysis for periodic structure, North-Holland, Amsterdam (1978)

[2] E. Sanchez Palencia, Nonhomogeneous media and vibration theory, Lectures notes in Physics, 127, Springer, Berlin (1980)

[3] J.L. Auriault, C. Boutin and C. Geindreau : Homogenization of Coupled Phenomena in Heterogenous Media, ISTE and Wiley (2009)

[4] T. Levy, Propagation of waves in a fluid-saturated porous elastic solid, Int. J. Engng Sci. 17 (1979) 1005-1014

[5] J.L. Auriault, Dynamic behaviour of a porous medium saturated by a newtonian fluid, Int. J. Engng Sci. 18 (1980) 775-785

[6] M.Y. Zhou, P. Sheng, First principles calculations of dynamic permeability in porous media, Phys. Rev. B 39 (1989) 12027-12039.

[7] D.M.J. Smeulders, R.L.G.M. Eggels, M.E.H. van Dongen, Dynamic permeability: reformulation of theory and new experimental and numerical data, J. Fluid Mech. 245 (1992) 211-227

[8] R. Burridge \& J.B. Keller, Poroelasticity equations derived from microstructure, J. Acoust. Soc. Am. 70 (1981) 1140â€"-1146

[9] A.N. Norris, On the viscodynamic operator in Biot's equations of poroelasticity, J. Wave Mat. Interact. 1 (1986) 365-380.

[10] D. Lafarge, P. Lemarinier, J.F. Allard, V. Tarnow, Dynamic compressibility of air in porous structures at audible frequencies, J. Acoust. Soc. Am. 102 (1997) 1995-2006.

[11] C. Boutin, Acoustics of rigid porous media with inner resonators, submitted to J. Acoust. Soc. Am. (2012)

\footnotetext{
16 Looking at the calculation made in the frequency domain, the figure 3 of this paper exhibits a ratio between the $P L$ description and the $J A$ one $\left(q=q^{\prime}=1\right)$ that is almost independent of frequency: this is the attenuation-without-distortion effect that is described by Eq. (59) in our wavenumber-based asymptotic calculation.
} 
[12] D. Lafarge, N. Nemati, Nonlocal theory of sound propagation in homogeneous rigid-framed porous media, submitted to Wave Motion (2012)

[13] R.V. Craster, J. Kaplunov, A.V. Pishugin, High-frequency homogenization for periodic media, Proc. R. Soc. A 466 (2010) 2341-2362

[14] R.V. Craster, J. Kaplunov, J. Postnova, High-Frequency Asymptotics, Homogenization and Localization for Lattices, Q. J. Mechanics Appl. Math. 63 (2010) 497-519

[15] R.V. Craster et al., Bloch dispersion and high frequency homogenization for separable doubly-periodic structures, Wave Motion 49 (2012) 333-346

[16] C. Boutin, A. Rallu, S. Hans, Large scale modulation of high frequency acoustic waves in periodic porous media, to appear in J. Acoust. Soc. Am. (2012)

[17] D.L. Johnson, J. Koplik, R. Dashen, Theory of dynamic permeability and tortuosity in fluid-saturated porous media, J. Fluid Mech. 176 (1987) 379402.

[18] Y. Champoux, J.F. Allard, Dynamic tortuosity and bulk modulus in airsaturated porous media, J. Appl. Phys. 70 (1991) 1975-1979.

[19] H. Darcy, Les fontaines publiques de la ville de Dijon, V. Dalmont, Paris, (1856)

[20] D. Lafarge, Sound propagation in porous materials having a rigid frame saturated by gas, (in French). Ph.D. Dissertation, Université du Maine, 1993.

[21] D.K. Wilson, Relaxation-matched modeling of propagation through porous media, including fractal structure, J. Acoust. Soc. Am. 94 (1993) 11361145 .

[22] D.K. Wilson, Simple, relaxational models for the acoustical properties of porous media, Appl. Acoust. 50 (1997) 171-188.

[23] S.R. Pride, F.D. Morgan, A.F. Gangi, Drag forces of a porous-medium acoustics, Phys. Rev. B (1993) 4964-4978.

[24] A. Cortis, Dynamic acoustic parameters of porous media: a theoretical, numerical and experimental investigation. Ph.D. Thesis, Delft University Press, 2002

[25] Z.E.A. Fellah, C. Depollier, M. Fellah, W. Lauriks, J.-Y. Chapelon, Influence of dynamic tortuosity and compressibility on the propagation of transient waves in porous media, Wave motion 41 (2005) 145-161.

[26] B.D. Plyushchenkov and T. I. Turchaninov, Acoustic logging modeling by refined Biot's equations, Int. J. Mod. Phys. C, 11 (2000) 365-396.

[27] D.K. Wilson, V.E. Ostashev, and S.L. Collier, Time-domain equations for sound propagation in rigid frame porous media, J. Acoust. Soc. Am. 116 (2004) 1889â€“-1892. 
[28] D.K. Wilson, S.L. Collier, V.E. Ostashev, D.F. Aldridge, N.P. Symons, and D. H. Marlin, Time-domain modelling of the acoustic impedance of porous surfaces, Acta. Acust. Acust. 92 (2005) 965â€"-975.

[29] D.K. Wilson, V.E. Ostashev, S.L. Collier, N.P. Symons, D.F. Aldridge, and D.H. Marlin, Time-domain calculations of sound interactions with outdoor ground surfaces, Appl. Acoust. 68 (2007) 173â€“-200.

[30] O. Umnova and D. Turo, Time domain formulation of the equivalent fluid model for rigid porous media (L), J. Acoust. Soc. Am. 125 (2009) 1860-1863

[31] Z.E.A. Fellah, M. Fellah, W. Lauriks, C. Depollier, J.Y. Chapelon, and Y.C. Angel, Solution in time domain of ultrasonic propagation equation in a porous material, Wave Motion 38 (2003) 151-163

[32] Z.E.A. Fellah, M. Fellah, W. Lauriks, and C. Depollier, Direct and inverse scattering of transient acoustic waves by a slab of rigid porous material, J. Acoust. Soc. Am. 113 (2003) 61â€"-72.

[33] Z.E.A. Fellah, A. Wirgin, M. Fellah, N. Sebaa, C. Depollier and W. Lauriks, A time-domain model of transient acoustic wave propagation in doublelayered porous media, J. Acoust. Soc. Am. 118 (2005) 661-670.

[34] Z.E.A. Fellah, M. Fellah, F.G. Mitri, N. Sebaa, W. Lauriks and C. Depollier, Transient acoustic wave propagation in air-saturated porous media at low frequencies, J. Appl. Phys. 102 (2007) 84906-84915

[35] G. Chiavassa, B. Lombard, Time domain numerical modeling of wave propagation in 2D heterogeneous porous media, Journal of Computational Physics, 230 (2011), 5288-5309.

[36] G. Lefeuve-Mesgouez, A. Mesgouez, G. Chiavassa, B. Lombard, Semianalytical and numerical methods for computing transient waves in $2 \mathrm{D}$ acoustic / poroelastic stratified media, Wave Motion 49 (2012), 667-680.

[37] G. Chiavassa, B. Lombard, Wave propagation across acoustic / Biot's media: a finite-difference method, Communications in Computational Physics 13 (2013), 985-1012.

[38] E. Blanc, G. Chiavassa, B. Lombard, Biot-JKD model: simulation of 1D transient poroelastic waves with fractional derivatives, to appear in Journal of Computational Physics (2013).

[39] J.D. Polack, X. Meynial, J. Kergomard, C. Cosnard and M. Bruneau, Reflection function of a plane sound wave in a cylindrical tube, Revue Phys. Appl. 22 (1987) 331-337.

[40] J.F. Allard and N. Atalla, Propagation of Sound in Porous Media: Modelling Sound Absorbing Materials, Second Edition, John Wiley \& Sons, 2009.

[41] D. Lafarge, The equivalent fluid model, in Materials and Acoustics Handbook, M. Bruneau and C. Potel eds, ISTE-Wiley, London, 2009, pp 205228. 
[42] L.D. Landau, E. Lifshitz, Electrodynamics of continuous media, Elsevier Butterworth-Heinemann, 2006.

[43] A. Cortis, D. Smeulders, J.L. Guermond, D. Lafarge, Influence of pore roughness on high-frequency permeability, Phys. Fluids 15 (2003) 17661775.

[44] R. Brown, Connection between the formation factor for electrical resistivity and fluid solid coupling factors in Biot's equations for acoustic waves in fluid-filled porous media, Geophysics 45 (1980) 1269-1275.

[45] M. Avellaneda, S. Torquato, Rigorous link between fluid permeability, electrical conductivity, and relaxation times for transport in porous media, Phys. Fluids A 3 (1991) 2529-2540.

[46] D. Lafarge, Comments on 'Rigorous link between fluid permeability, electrical conductivity, and relaxation times for transport in porous media', Phys. Fluids A 5 (1992) 500-503.

[47] D.L. Johnson, J. Koplik, L.M. Schwartz, New pore-size parameter characterizing transport in porous media, Phys. Rev. Lett. 57 (1986) 2564-2567.

[48] J.L. Auriault, L. Borne, R. Chambon, Dynamics of porous saturated media, checking of the generalized law of Darcy, J. Acoust. Soc. Am. 77 (1985) 1641-1650.

[49] S.R. Pride, Governing equations for the coupled electromagnetics and acoustics of porous media, Phys. Rev. B50 (1994) 15678-15696

[50] S. Torquato, Relationship between permeability and diffusion-controlled trapping constant of porous media, Phys. Rev. Lett. 64 (1990) 2644-2646.

[51] C. Boutin, C. Geindreau, Estimates and bounds of dynamic permeability of granular media, J. Acoust. Soc. Am. 124 (2008), 3576-3593.

[52] C. Zwikker, C.W. Kosten, Sound Absorbing Materials, Elsevier, New York, 1949.

[53] G. Kirchhoff, Uber des Einfluss der Wärmeleitung in einem Gase auf die Schallbewegung, Annalen der Physik and Chemie 134 (1868) 177-193.

[54] J. Kergomard, J.D. Polack, J. Gilbert, Propagation speed of a plane impulsive wave in a sound tube (in French), J. Acoustique 4 (1991),467-483.

[55] H. Tijdeman, On the propagation of sound waves in cylindrical tubes, J. Sound Vib. 39 (1975) 1-33.

[56] D.H. Keefe, Acoustical wave propagation in cylindrical ducts: Transmission line parameter approximations for isothermal and nonisothermal boundary conditions, J. Acoust. Soc. Am. 75 (1984), 58-62.

[57] L.D. Landau, E. Lifshitz, Fluid Mechanics, Vol. 6 (2nd ed.), ButterworthHeinemann, 1987. 